\title{
The role of initial chest X-ray in triaging patients with suspected COVID-19 during the pandemic
}

\author{
Hyunjoong W. Kim ${ }^{1}$ (D) K. M. Capaccione ${ }^{1} \cdot$ Gen Li $^{2} \cdot$ Lyndon Luk $^{1} \cdot$ Reginald S. Widemon ${ }^{1} \cdot$ Ozair Rahman ${ }^{1}$. \\ Volkan Beylergil $^{1}$ - Ryan Mitchell ${ }^{1}$ • Belinda M. D'Souza ${ }^{1}$ - Jay S. Leb ${ }^{1}$ - Shifali Dumeer ${ }^{1}$. Stuart Bentley-Hibbert ${ }^{1}$. \\ Michael Liu ${ }^{1} \cdot$ Sachin Jambawalikar ${ }^{1}$. John H. M. Austin ${ }^{1}$ - Mary Salvatore ${ }^{1}$
}

Received: 13 April 2020 / Accepted: 12 June 2020 / Published online: 22 June 2020

(C) American Society of Emergency Radiology 2020

\begin{abstract}
Purpose The purpose of our research is to evaluate the usefulness of chest X-ray for triaging patients with suspected COVID-19 infection.

Methods IRB approval was obtained to allow a retrospective review of adult patients who presented to the Emergency Department with a complaint of fever, cough, dyspnea or hypoxia and had a chest X-ray between 12 March 2020 and 26 March 2020. The initial chest X-ray was graded on a scale of $0-3$ with grade 0 representing no alveolar opacities, grade $1:<1 / 3$ alveolar opacities of the lung, Grade 2: 1/3 to 2/3 lung with alveolar opacities and grade $3:>2 / 3$ alveolar opacities of the lung. Past medical history of diabetes and hypertension, initial oxygen saturation, COVID-19 testing results, intubation, and outcome were also collected.

Results Four hundred ten patient chest X-rays were reviewed. Oxygen saturation and X-ray grade were both significantly associated with the length of stay in hospital, the hazard ratio (HR) of discharge was 1.05 (95\% CI [1.01, 1.09], $p=0.017)$ and $0.61(95 \% \mathrm{CI}[0.51,0.73], p<0.001)$, respectively. In addition, oxygen saturation and X-ray grade were significant predictors of intubation (odds ratio (OR) of intubation is $0.88(95 \% \mathrm{CI}[0.81,0.96], p=0.004)$ and $3.69(95 \% \mathrm{CI}$ $[2.25,6.07], p<0.001)$.

Conclusions Initial chest $\mathrm{X}$-ray is a useful tool for triaging those subjects who might have poor outcomes with suspected COVID19 infection and benefit most from hospitalization.
\end{abstract}

Keywords COVID-19 $\cdot$ SARS-CoV-2 $\cdot$ Coronavirus $\cdot$ Chest $\cdot$ X-ray $\cdot$ CXR

\section{Introduction}

COVID-19 is the name of the disease caused by the novel coronavirus (SARS-CoV-2) that originated from Wuhan,

\section{Highlights}

- Patients with lower degree of alveolar opacities on chest X-ray have shorter length of stay.

- Patients with higher degree of alveolar opacities on chest X-ray are more likely to get intubated.

- Patients who are older tend to have higher degree of alveolar opacities on chest X-ray.

- Older people have significantly higher risk of death.

Hyunjoong W. Kim

hyk9023@nyp.org

1 Department of Radiology, Columbia University Irving Medical Center, 622 W 168th Street, New York, NY 10032, USA

2 Department of Biostatistics, Columbia University, New York, NY, USA
China in December 2019 [1]. Since then, there have been numerous cases outside of China, as evidenced by a declaration of "public health emergency of international concern" by the World Health Organization (WHO) on 30 January 2020. The USA has seen a significant increase in positive COVID19 cases since its first documented positive patient on 20 January 2020 and currently has over 475,000 cases nationwide as of 10 April 2020, with New York City accounting for over 92,000 cases. WHO declared COVID-19 as a pandemic on 11 March 2020.

The COVID-19 pandemic is not the first pandemic of the twenty-first century, yet it is the most impactful due to the relative ease of transmission, number of victims, and death rate globally. Alonso-Zaldivar described the ubiquity of the disease with 3 out of 4 US hospitals caring for COVID-19 patients. Many patients are treated as if they are infected because of the uncertainty of available testing procedures [2]. Access to diagnostic PCR testing was initially limited because of a short supply of testing kits; only patients whose treatment 
would be altered based on the results or those at high risk for a poor clinical outcome were tested [3].

Chest X-rays are ordered for most patients who present to the emergency room with symptoms suggestive of COVID-19 and have been used as a surrogate marker for disease in lieu of adequate PCR testing. The classic appearance on chest X-ray is lower lobe predominant alveolar opacities often with superimposed atelectasis [4]. However, chest radiographs are limited for diagnosis in that they may be normal in early or mild disease [5]. The purpose of the present study is to see if initial chest radiographs in the Emergency Department (ED) of a large New York City hospital located in northern Manhattan could be beneficial in triaging patients with suspected COVID-19 infection.

\section{Methods}

IRB approval was obtained to allow a retrospective review of adult patients (age 21 or older) who presented to the ED with a complaint of fever, cough, dyspnea, or hypoxia and had an initial chest radiograph between 12 March 2020 and 26 March 2020. Waiver of informed consent was approved by the institution. Data was stored on Box (Box, Inc., Redwood City, CA, USA), which is a HIPAA-compliant cloud storage system. Initial data set was extracted with $\mathrm{M}^{*}$ Modal Catalyst (M*Modal, Pittsburgh, PA, USA), an advanced query software, of ED chest radiographs with clinical indication of "fever," "cough," "dyspnea," and "hypoxia." These key words were chosen to widely capture patients with suspected COVID-19 symptoms during the initial pandemic when there was shortage of laboratory testing. One thousand two hundred fifty-six patients satisfied these criteria and a random sample of 416 was taken to be analyzed. The patients' medical records were reviewed and age, gender and initial oxygen saturation were documented. Also, note was made of history of diabetes and hypertension. If performed, the patient's SARS-CoV-2 status was recorded along with patient outcome (discharged from ED, admission, or death). The chest radiographs were reviewed by an experienced thoracic radiologist with over 20 years of experience and graded on a scale of $0-3$ with grade 0 representing no alveolar opacities, grade $1:<1 / 3$ alveolar opacities, grade $2: 1 / 3$ to $2 / 3$ alveolar opacities, and grade $3:>$ 2/3 alveolar opacities (Fig. 1).

Cox proportional hazards models (for time to event data), proportional odds models (for ordinal categorical data), and logistic regression models (for binary data) were used for association analysis. $p$ values $<0.05$ were considered statistically significant. Statistical analysis was performed using R (version 3.6.2, R Core Team, Vienna, Austria).

\section{Results}

One thousand two hundred fifty-six patients presented to the CUIMC ED with complaint of fever, cough, shortness of breath, or hypoxia and had a chest X-ray between 12 March 2020 and 26 March 2020. From this, a random
Fig. 1 Visual representation of the grading system for the chest radiographs, in which a grade 0 assigned for normal, $\mathbf{b}$ grade 1 assigned for alveolar opacities $<1 / 3$ of lung, $\mathbf{c}$ grade 2 assigned for alveolar opacities $1 / 3$ to $2 / 3$ of lung, and $\mathbf{d}$ grade 3 assigned for alveolar opacities $>2 / 3$ of lung
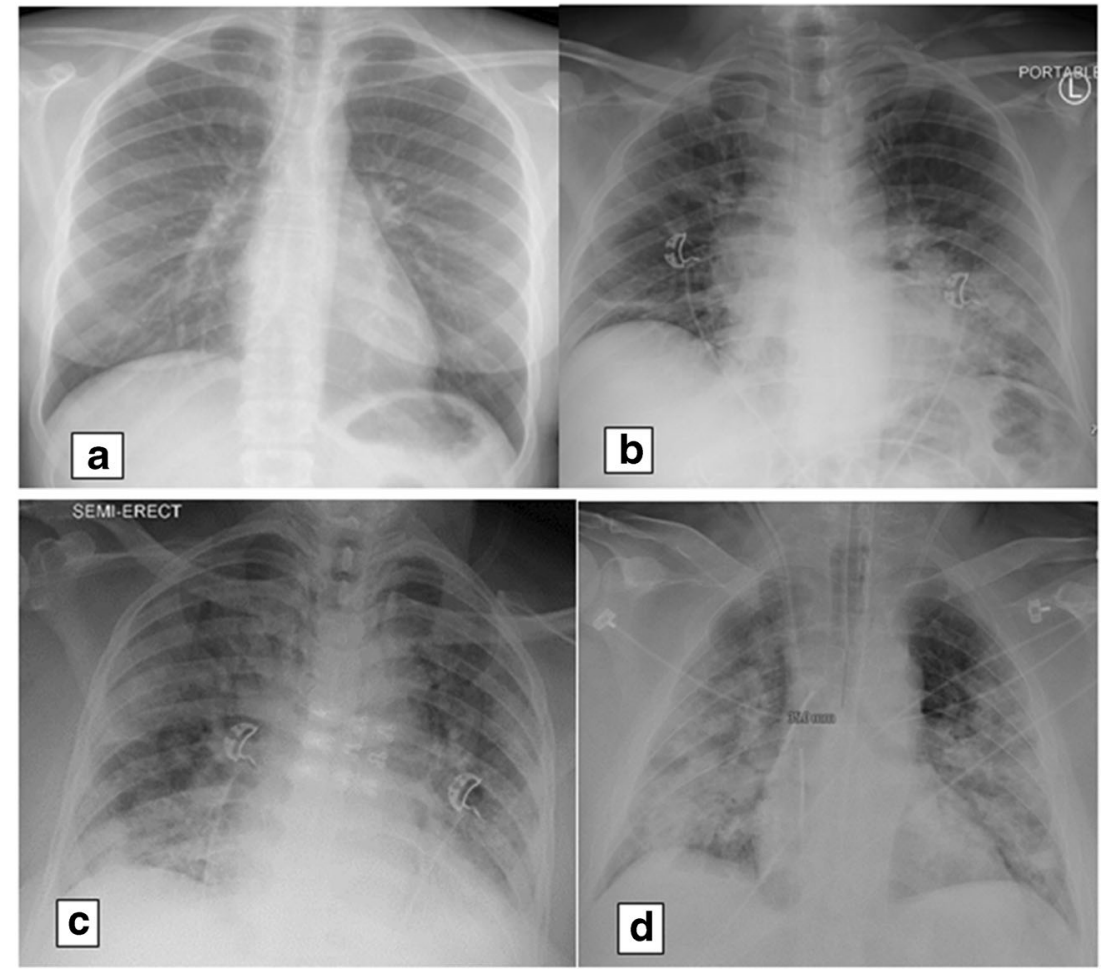
Table 1 Patient demographics and outcomes based on initial chest X-ray findings of patients suspected of having COVID-19 infection

\begin{tabular}{lllll}
\hline & Grade $0^{\mathrm{a}}$ & Grade $^{\mathrm{b}}$ & Grade $^{\mathrm{c}}$ & Grade $^{\mathrm{d}}$ \\
\hline \# of patients & $226 / 410(55 \%)$ & $140 / 410(34 \%)$ & $34 / 410(8 \%)$ & $10 / 410(2 \%)$ \\
Average age & 55 & 63 & 63 & 72 \\
Gender (\% female) & $112 / 226(50 \%)$ & $64 / 140(46 \%)$ & $20 / 34(59 \%)$ & $8 / 10(80 \%)$ \\
SARS-CoV-2 positive & $36 / 226(16 \%)$ & $61 / 140(44 \%)$ & $24 / 34(71 \%)$ & $7 / 10(70 \%)$ \\
Not tested for SARS-CoV-2 & $167 / 226(74 \%)$ & $52 / 140(37 \%)$ & $4 / 34(12 \%)$ & $1 / 10(10 \%)$ \\
Average initial \% O2 saturation & 97 & 96 & 95 & 92 \\
Hypertension & $100 / 226(44 \%)$ & $76 / 140(54 \%)$ & $22 / 34(65 \%)$ & $7 / 10(70 \%)$ \\
Diabetes & $43 / 226(19 \%)$ & $45 / 140(32 \%)$ & $12 / 34(35 \%)$ & $2 / 10(20 \%)$ \\
Discharged from ED & $172 / 226(76 \%)$ & $64 / 140(46 \%)$ & $4 / 34(12 \%)$ & $0 / 10(0 \%)$ \\
Intubated & $2 / 226(<1 \%)$ & $15 / 140(11 \%)$ & $10 / 34(29 \%)$ & $5 / 10(50 \%)$ \\
Hospitalization $>10$ days & $3 / 226(1 \%)$ & $12 / 140(9 \%)$ & $8 / 34(24 \%)$ & $5 / 10(50 \%)$ \\
Death & $2 / 226(<1 \%)$ & $9 / 140(6 \%)$ & $6 / 34(18 \%)$ & $3 / 10(30 \%)$ \\
\hline
\end{tabular}

${ }^{\text {a }}$ Grade 0: normal chest X-ray

${ }^{\mathrm{b}}$ Grade $1:<1 / 3$ alveolar opacities on chest X-ray

${ }^{\mathrm{c}}$ Grade 2: 1/3-2/3 alveolar opacities on chest X-ray

${ }^{\mathrm{d}}$ Grade $3:>2 / 3$ alveolar opacities on chest X-ray sample of 416 patients made up the study population. Six patients were excluded because they had another reason for shortness of breath, cough or fever which was not related to COVID-19 infection. Three patients had prior history of pulmonary disease, such as interstitial lung disease, bronchiectasis, and scarring that would have confounded the grading for alveolar opacities. Two patients had pulmonary edema from cardiac issues, such as acute on chronic congestive heart failure or aortic insufficiency. One patient presented due to physical assault and clinically had no concerns regarding potential COVID-19 infection. No other patients were excluded and there were no self-discharges from the ED. The final cohort consisted of 410 initial chest $\mathrm{X}$-rays.

The average age was $59.50 \%$ of patients were female. The average initial oxygen saturation was $96 \%$; of note, most were measured on room air or at patient's baseline. $25 \%$ had diabetes and 50\% had hypertension. 55\% were not tested for COVID-19 with a PCR exam. Of those who were tested, $69 \%$ were positive. Overall, 32 patients were intubated on average of 3.8 days after hospital admission. Twenty patients

Table 2 Factors associated with time to discharge from hospital

\begin{tabular}{lllr}
\hline & Hazard ratio & $95 \%$ CI & $p$ value \\
\hline Age & 0.9946 & $0.9871-1.0023$ & 0.1672 \\
Gender (male) & 1.0250 & $0.8268-1.2707$ & 0.8219 \\
Oxygen saturation & 1.0509 & $1.0089-1.0946$ & 0.0170 \\
Diabetes & 1.0870 & $0.8297-1.4241$ & 0.5448 \\
Hypertension & 0.9086 & $0.7114-1.1605$ & 0.4426 \\
Chest X-ray grade & 0.6125 & $0.5134-0.7307$ & $<0.001$ \\
\hline
\end{tabular}

died, on average 7.8 days after admission. Fifty-nine percent of patients were discharged from the ED. Twenty-five patients remained hospitalized at the time of this manuscript with an average of 19 days since admission. Demographics and clinical outcomes of patients who presented to the ED are divided based on the initial chest X-ray grading in Table 1.

Excluding the patients who died, 390 surviving patients were analyzed. Oxygen saturation and X-ray grade are both significantly associated with the length of stay in hospital. The HR of discharge is 1.05 (95\% CI [1.01, 1.09], $p=0.017$ ) with one unit increase in $\mathrm{O} 2$ saturation, and 0.61 (95\% CI [0.51, $0.73], p<0.001$ ) with one grade higher for $\mathrm{X}$-ray (Table 2 ). Oxygen saturation and $\mathrm{X}$-ray grade were significant predictors of intubation. The odds ratio (OR) of intubation was 0.88 (95\% CI [0.81, 0.96], $p=0.004$ ) with one unit increase in $\mathrm{O} 2$ saturation, and 3.69 (95\% CI [2.25, 6.07], $p<0.001)$ with one grade higher for X-ray (Table 3 ).

The hazard ratio (HR) of death with one-year increase in age is 1.095 (95\% confidence interval [1.041, 1.152], $p=$ 0.0004) (Table 4). Figure 2 is Kaplan-Meier curve for time to death for 410 patients.
Table 3 Factors associated with likelihood of intubation

\begin{tabular}{llll}
\hline & Odds ratio & $95 \%$ CI & $p$ value \\
\hline Age & 1.0063 & $0.9765-1.0371$ & 0.6809 \\
Gender (male) & 1.2238 & $0.5176-2.8933$ & 0.6455 \\
Oxygen saturation & 0.8810 & $0.8087-0.9598$ & 0.0037 \\
Diabetes & 1.4063 & $0.5648-3.5023$ & 0.4640 \\
Hypertension & 1.1079 & $0.4195-2.9256$ & 0.8362 \\
Chest X-ray grade & 3.6927 & $2.2459-6.0715$ & $<0.001$ \\
\hline
\end{tabular}


Table 4 Factors associated with increased likelihood of death

\begin{tabular}{llll}
\hline & Hazard ratio & $95 \%$ CI & $p$ value \\
\hline Age & 1.0950 & $1.0409-1.1520$ & 0.0004 \\
Gender (male) & 0.7017 & $0.2668-1.8451$ & 0.4726 \\
Oxygen saturation & 1.0080 & $0.8970-1.1327$ & 0.8935 \\
Diabetes & 1.3263 & $0.5163-3.4124$ & 0.5567 \\
Hypertension & 5.0263 & $0.6471-39.0408$ & 0.1226 \\
Chest X-ray grade & 1.4534 & $0.8328-2.5365$ & 0.1882 \\
\hline
\end{tabular}

\section{Discussion}

Chest X-rays were performed on all ED patients as part of triaging those who were most in need of hospitalization. Regardless of chest X-ray result, all patients were counseled to isolate themselves from others to slow the spread of infection and dampen the curve with the hope of decreasing hospitalizations and need for ventilators $[6,7]$.

The majority of patients (55\%) who presented to ED with symptoms suspicious for COVID-19 infection had a normal chest X-ray. They were on average younger than those who had consolidations on chest X-ray. The majority of these patients $(74 \%)$ were not tested for SARS-CoV-2 and had higher average initial oxygen saturation compared to those that tested positive. There were fewer patients with diabetes and hypertension in this subgroup; diabetes and hypertension have been associated with worse outcomes for patients with COVID-19 infection $[8,9]$. The majority of these patients $(76 \%)$ were discharged from the ED, with only $1 \%$ having a prolonged hospital stay of 10 days or greater. Less than $1 \%$ died or were intubated.

However, 24\% of patients with a normal chest radiograph initially was not discharged from the ED and was admitted for further treatment. A normal chest radiograph alone cannot be used for triage of COVID-19, as mild or early disease may not be reflected radiographically, as radiographic findings may lag behind clinical presentation.

In contrast, those who had findings of alveolar opacities on chest X-ray were older than those without alveolar opacities. Increasing severity of alveolar opacities on chest X-ray was associated with increasing age. Surprisingly, the percentage of females was higher in the group with the highest chest X-ray grade. Previous reports had described a worse prognosis for men, which were explained by greater likelihood of smoking $[10,11]$. This group of patients was more likely to have a PCR test and to test positive. As would be expected, their oxygen saturations were lower proportional to the amount of disease. The majority of the patients in this group had hypertension and diabetes, which has been reported $[8,9]$. These patients had worse outcomes overall and proportionate to the amount of consolidation. Fewer could be discharged from emergency room and a greater percentage required intubation and ultimately died. Not surprisingly, lower oxygen saturation and higher degree of alveolar opacities on the initial chest X-ray regardless of COVID-19 result were associated with increased duration of hospitalization and increased likelihood of intubation.

The Fleischner Society published a multinational consensus statement on 7 April 2020 regarding the use of chest imaging during the COVID-19 pandemic, which was published after the data collection period for this paper, and presented three different scenarios and algorithm for recommendation on the use of chest imaging. Essentially, the early period of the COVID-19 pandemic in New York City mirrored the third scenario depicted in the consensus statement, which was the lack of availability of COVID-19 testing. The consesus statement reiterated the importance of imaging to quickly triage patients when presented with high influx of new patients [12].

Limitations include a retrospective, single center study with a single radiologist interpreting the radiographs to a novel grading system tailored to the current pandemic within the
Fig. 2 Overall survival curve for 410 patients

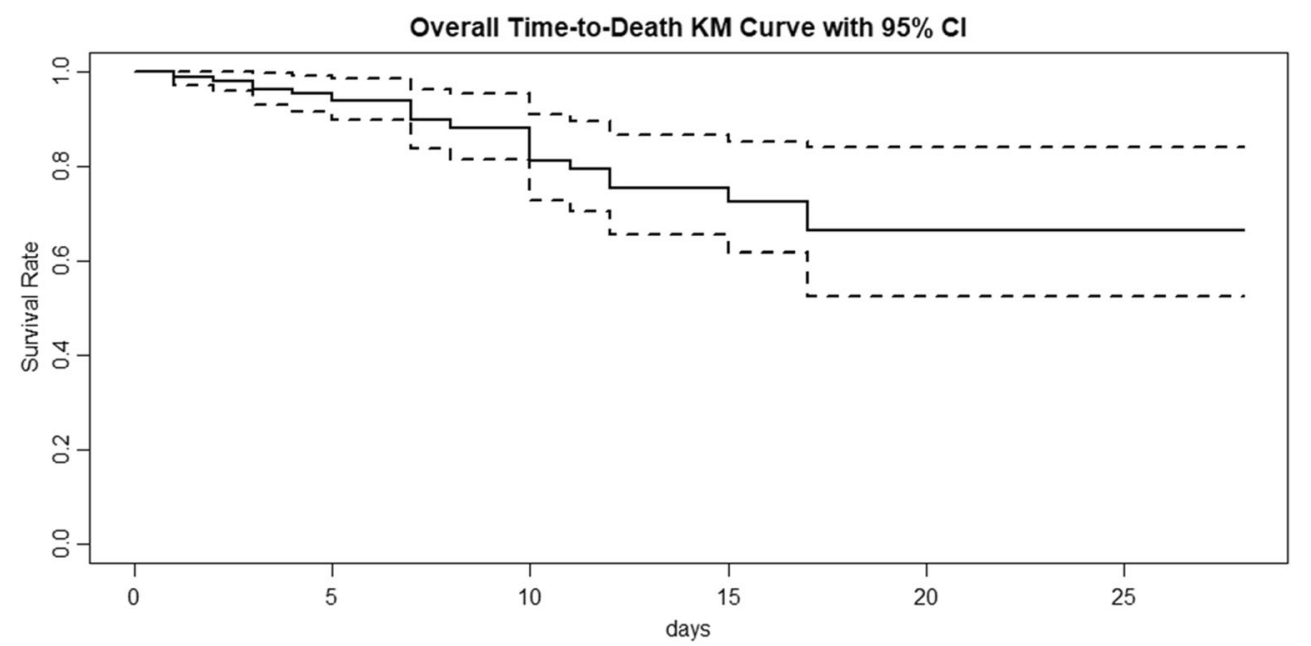


United States. Future studies should include more readers to compare performance and can be extended to include multiple institutions treating a large number of patients with COVID19. Many patients remain hospitalized in critical condition and on mechanical ventilation; the data presented in this manuscript are related to the current state of our institution during the pandemic and are subject to change as patients recover or pass away.

\section{Conclusion}

In conclusion, a normal chest $\mathrm{X}$-ray does not exclude the diagnosis of COVID-19, but can serve as a useful diagnostic tool for evaluation and appropriate triage of patients with suspected COVID-19 infection, particularly at a time when hospital beds are limited, demand is high, and the disease begins to affect different regions of the country that may not have suitable inpatient and ICU bed capacity. Patients with near total or complete involvement of the lungs are at higher risk for poor clinical outcome with higher rates of intubation and extended hospitalization.

\section{Code availability Not applicable.}

\section{Compliance with ethical standards}

Conflict of interest Mary Salvatore, MD is a speaker and consultant for Genentech and Boehringer Ingelheim, and received grant funding from Genentech, Boehringer Ingelheim, and Lunglife AI.

Ethics approval This study was conducted retrospectively from data obtained for clinical purposes. The study received Institutional Review Board approval.

Consent to participate A waiver of informed consent was granted for this retrospective study.

Consent to publish The included images are of X-rays without any identifying information and not necessary to obtain consent.

\section{References}

1. Li Q, Guan X, Wu P, Wang X, Zhou L, Tong Y, Ren R, Leung KSM, Lau EHY, Wong JY, Xing X, Xiang N, Wu Y, Li C, Chen Q, Li D, Liu T, Zhao J, Liu M, Tu W, Chen C, Jin L, Yang R, Wang Q, Zhou S, Wang R, Liu H, Luo Y, Liu Y, Shao G, Li H, Tao Z, Yang Y, Deng Z, Liu B, Ma Z, Zhang Y, Shi G, Lam TTY, Wu JT, Gao GF, Cowling BJ, Yang B, Leung GM, Feng Z (2020) Early transmission dynamics in Wuhan, China, of novel coronavirus-infected pneumonia. N Engl J Med 382:1199-1207

2. Alonso-Zaldivar R. With Worst Expected to Come, 3 out of 4 U.S. Hospitals Are Treating Patients with COVID-19. Fortune. Published on line April 6, 2020

3. Hadaya J, Schumm M, Livingston EH (2020) Testing individuals for coronavirus disease 2019 (COVID-19). JAMA

4. Rodrigues JCL, Hare SS, Edey A, Devaraj A, Jacob J, Johnstone A, McStay R, Nair A, Robinson G (2020) An update on COVID-19 for the radiologist - a British Society of Thoracic Imaging statement. Clin Radiol 75(5):323-325

5. Wong HYF, Lam HYS, Fong AH, et al (2020) Frequency and distribution of chest radiographic findings in COVID-19 positive patients. Radiology

6. Koo JR, Cook AR, Park M, et al (2020) Interventions to mitigate early spread of SARS-CoV-2 in Singapore: a modelling study. Lancet Infect Dis

7. Fauci AS, Lane HC, Redfield RR (2020) Covid-19-navigating the uncharted. N Engl J Med 382:1268-1269

8. Bloomgarden ZT (2020) Diabetes and COVID-19. J Diabetes 12: 347-349

9. Zheng Y, Ma Y, Zhang J et al (2020) COVID-19 and the cardiovascular system. Nat Rev Cardiol 17:259-260. https://doi.org/10. 1038/s41569-020-0360-5

10. Liu S, Zhang M, Yang L, Li Y, Wang L, Huang Z, Wang L, Chen Z, Zhou M (2017) Prevalence and patterns of tobacco smoking among Chinese adult men and women: findings of the 2010 national smoking survey. J Epidemiol Community Health 71:154-161

11. Chen N, Zhou M, Dong X, Qu J, Gong F, Han Y, Qiu Y, Wang J, Liu Y, Wei Y, Xia J', Yu T, Zhang X, Zhang L (2020) Epidemiological and clinical characteristics of 99 cases of 2019 novel coronavirus pneumonia in Wuhan, China: a descriptive study. Lancet 395:507-513

12. Rubin GD, Ryerson CJ, Haramati LB et al (2020) The role of chest imaging in patient management during the COVID-19 pandemic: a multinational consensus statement from the Fleischner society. Radiology 7:201365

Publisher's note Springer Nature remains neutral with regard to jurisdictional claims in published maps and institutional affiliations. 